\title{
Universal behaviour of silica suspensions gelled under shear*
}

\author{
H J M Hanley ${ }^{1,2}$, C D Muzny ${ }^{3}$, J Bartlett $^{1}$ and E Drabarek ${ }^{1}$ \\ ${ }^{1}$ Australian Nuclear Science and Technology Organisation, PMB 1, Menai, NSW 2234, Australia \\ 2 Australian National University, Canberra, ACT 0200, Australia \\ ${ }^{3}$ Physical and Chemical Properties Division, National Institute of Standards and Technology, \\ Boulder, CO 80305, USA
}

Received 15 February 2007, in final form 6 July 2007

Published 13 September 2007

Online at stacks.iop.org/JPhysCM/19/416108

\begin{abstract}
The shear-influenced gelation of three aqueous suspensions consisting of silica particles of nominal diameter 7, 12 and $24 \mathrm{~nm}$, respectively, is reported. It is shown that the viscosity/stress of a gelling system increases with time after gel initiation, reaches a maximum, then falls to a plateau value. A very simple relation between this maximum stress and the precursor volume fraction is verified experimentally.
\end{abstract}

(Some figures in this article are in colour only in the electronic version)

A few years ago we discussed how an applied shear affects the gelation process in a dispersion of colloidal silica $[1,2]$. As reported in that work we initiated gelation in a given suspension of volume fraction, $\phi$, subjected the system to a steady shear rate, $\gamma$, and recorded the viscosity/stress as a function of time. In the limit when the shear rate approached zero the viscosity, $\eta$, increased as the gelation advanced until, as expected, it became essentially infinite at a time defined loosely as the gel time, $t_{\mathrm{m}}$. At finite shear rate, however, we found that the final gel state was postponed; specifically, that the viscosity initially increased with time but then peaked at a time $\sim t_{\mathrm{m}}$ until finally decreasing to a plateau value, figure $1^{4}$. This relatively low viscosity state could be held indefinitely by maintaining the shear, but the system gelled once the shear was removed ${ }^{5}$. Some preliminary results from other systems gelling under shear hint strongly that the behaviour of the silica dispersion could be general [3].

The stress corresponding to the peak viscosity is a critical stress $\Pi_{c}$ which, for a given sample, appeared to be constant to within the experimental uncertainties over a wide range of applied shear rates, and was independent of the gel time, $t_{\mathrm{m}}$. An argument can be made,

\footnotetext{
* Publication in part of the National Institute of Standards and Technology, not subject to copyright.

4 Small angle neutron scattering and dynamic light scattering indicated that the drop in viscosity is explained by a structural densification of the growing clusters when the stress corresponding to the peak viscosity value is reached.

5 However, oscillatory experiments and the SANS studies indicate that this final gel has a short range order different from that of the gel formed in the absence of a shear. See [2].
} 


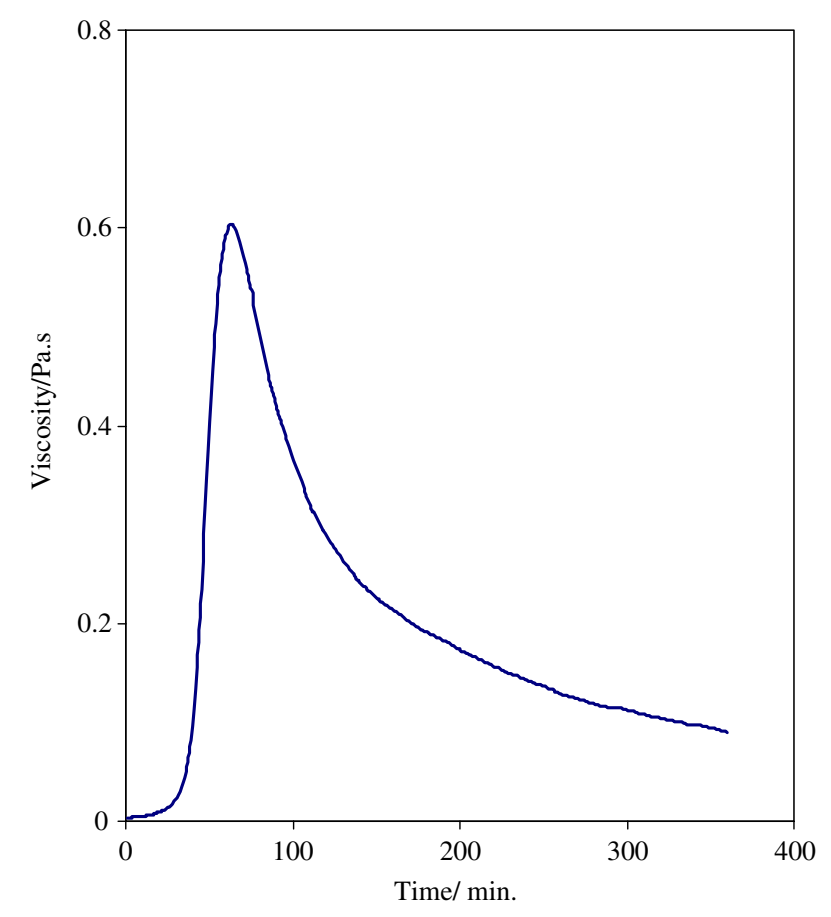

Figure 1. Plot of the viscosity behaviour of a gelling aqueous system of $12 \mathrm{~nm}$ colloidal silica particles subjected to a shear rate of $500 \mathrm{~s}^{-1}$. The maximum corresponds to the critical stress, $\Pi_{\mathrm{c}}$ discussed in the text.

therefore, that the critical stress is a universal parameter that depends to a first approximation on only the precursor volume fraction. In fact, a heuristic relationship between these variables is immediately apparent. Assume colloidal particles of radius $r_{\mathrm{p}}$ coalesce on gelation to form clusters of radius $R_{\mathrm{c}}$ with fractal dimension $d_{\mathrm{f}}$ at a volume fraction $\Phi$. Define a reduced length $\zeta=R_{\mathrm{c}} / r_{\mathrm{p}}$. If the primary particles remain unchanged during the process, we can write the standard relation between the volume fractions $[1,4]$ :

$$
\Phi \approx \phi \zeta^{3-d_{\mathrm{f}}}
$$

Provided the energy is constant during the gelling process which, as discussed in [1], is in practise true, we have by dimensional arguments that the stress $\Pi$ must vary as $\zeta^{d_{\mathrm{f}}-3}$. Hence

$$
\Pi \approx \phi / \Phi \text {. }
$$

But $\Phi$ tends to 1 as the stress approaches the critical value $\Pi_{c}$, since the gel spans the container. Whence

$$
\Pi_{c} / \phi \approx C
$$

where $C$ is a constant.

This short note reports a test of this expression. We gelled three precursor aqueous dispersions of colloidal $\mathrm{SiO}_{2}$ particles of nominal diameter 7, 12 and $24 \mathrm{~nm}$-corresponding to volume fractions of $0.170,0.228$ and 0.307 , respectively. Gelation was induced in a given dispersion by adding $\mathrm{HCl}$ following the procedure outlined in [1]. Immediately after gel initiation the dispersion was placed in a commercial Couette rheometer thermostatted to $298 \mathrm{~K}$, 


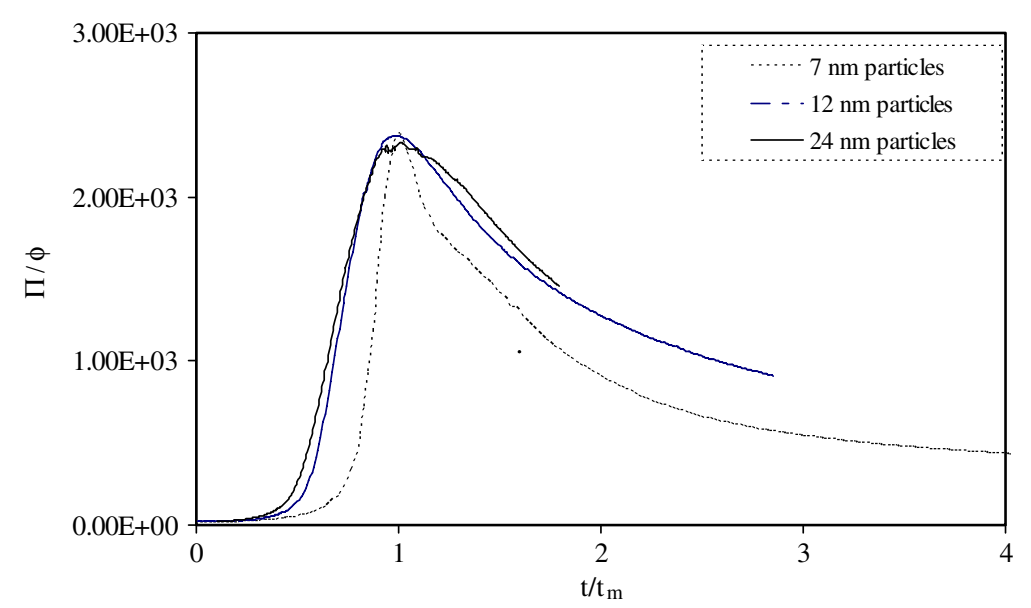

Figure 2. Test of equation (3) using three suspensions containing silica particles of diameter 7, 12 and $24 \mathrm{~nm}$, respectively. The systems were subjected to an applied shear of $1000 \mathrm{~s}^{-1}$. Shown is a plot of the term $\Pi / \phi$ as a function of reduced time. The term $\phi$ is the precursor suspension volume fraction and $t_{\mathrm{m}}$ is an effective gelation time for the static system.

kept at ambient pressure, and subjected to an applied shear. The stress and viscosity were recorded as a function of time, $t$. All systems were subjected to shear rates in the range $5 \leqslant \gamma / s^{-1} \leqslant 1600$. The majority of the runs were then repeated using a cone-on-plate rheometer.

The most complete comparative data set was obtained from the three systems subjected to the shear rate $\gamma=1000 \mathrm{~s}^{-1}$. Figure 2 depicts the evaluation of equation (3): shown is the plot of $\Pi_{\mathrm{c}} / \phi$ as a function of $t / t_{\mathrm{m}}$. Similar curves were obtained at shear rates of 200 and $500 \mathrm{~s}^{-1}$. For all runs, representing the three systems over the range of shear rates studied, we have the overall result that

$$
\begin{aligned}
& 10^{3}\left(\Pi_{c} / \phi\right)_{\text {couette }}=2.37 \pm 0.08 \\
& 10^{3}\left(\Pi_{c} / \phi\right)_{\text {cone-on-plate }}=2.34 \pm 0.25
\end{aligned}
$$

It is thus clear that equation (3) is justified. As figure (2) indicates, the curves deviate when $t / t_{\mathrm{m}} \neq 1$, since the equation requires that the cluster volume fraction be constant, but other approximations - including the very obvious simplification that the stress is independent of the shear-do not appear significant. In short, although the relation does not address any details of the gel structure, and changes to that structure due to a shear, the equation appears very robust and could well be system independent. We intend to investigate this hypothesis.

\section{References}

[1] Hanley H J M, Muzny C D, Butler B D, Straty G C, Bartlett J and Drabarek E 1999 J. Phys.: Condens. Matter 111369

[2] Drabarek E, Bartlett J, Hanley H J M, Woolfrey J L, Muzny C D and Butler B D 2000 J. Sol-Gel Sci. Technol. 19279

[3] Hanley H J M, Muzny C D, Bartlett J and Drabarek E, 2007 Studies of gelation in precipitating hydrocarbon mixtures, at press

[4] For instance, Dietler G, Aubert C, Cannell D S and Wiltzius P 1986 Phys. Rev. Lett. 573117

Wessel R and Ball R C 1992 Phys. Rev. A 46 R3008 